\title{
Salmonella transcriptional signature in Tetrahymena phagosomes and role of acid tolerance in passage through the protist
}

\author{
Marc Yi Ming Rehfuss, Craig Thomas Parker and Maria Theresa Brandl \\ Produce Safety and Microbiology Unit, Western Regional Research Center, Agricultural Research Service, \\ US Department of Agriculture, Albany, CA, USA
}

\begin{abstract}
Salmonella enterica Typhimurium remains undigested in the food vacuoles of the common protist, Tetrahymena. Contrary to its interaction with Acanthamoeba spp., $S$. Typhimurium is not cytotoxic to Tetrahymena and is egested as viable cells in its fecal pellets. Through microarray gene expression profiling we investigated the factors in $S$. Typhimurium that are involved in its resistance to digestion by Tetrahymena. The transcriptome of $S$. Typhimurium in Tetrahymena phagosomes showed that 989 and 1282 genes were altered in expression compared with that in water and in LB culture medium, respectively. A great proportion of the upregulated genes have a role in anaerobic metabolism and the use of alternate electron acceptors. Many genes required for survival and replication within macrophages and human epithelial cells also had increased expression in Tetrahymena, including $\mathrm{mgtC}$, one of the most highly induced genes in all three cells types. A $\Delta \mathrm{mgtC}$ mutant of $S$. Typhimurium did not show decreased viability in Tetrahymena, but paradoxically, was egested at a higher cell density than the wild type. The expression of adiA and adiY, which are involved in arginine-dependent acid resistance, also was increased in the protozoan phagosome. A $\triangle$ adiAY mutant had lower viability after passage through Tetrahymena, and a higher proportion of S. Typhimurium wild-type cells within pellets remained viable after exposure to $\mathrm{pH} 3.4$ as compared with uningested cells. Our results provide evidence that acid resistance has a role in the resistance of Salmonella to digestion by Tetrahymena and that passage through the protist confers physiological advantages relevant to its contamination cycle.
\end{abstract}

The ISME Journal (2011) 5, 262-273; doi:10.1038/ismej.2010.128; published online 5 August 2010

Subject Category: microbe-microbe and microbe-host interactions

Keywords: enteric pathogen; grazing; human pathogen; protozoa; resistance; vacuole

\section{Introduction}

Grazing by phagocytic protozoa is a major factor in shaping bacterial populations in aquatic, soil, and anthropogenic ecosystems (Pace, 1988; Barker and Brown, 1994). Bacteria that resist grazing by protozoa may show increased environmental fitness (Hahn and Hofle, 2001). Grazing resistance may occur through pre-ingestional adaptations involving development of oversized cells, surface masking or microcolony formation, and through post-ingestional adaptations that include development of toxin release, digestional resistance and/or intracellular growth (Matz and Kjelleberg, 2005). Indeed, the intracellular pathogens Mycobacterium avium, Chlamydia pneumoniae, Listeria monocytogenes and Legionella pneumophila replicate in the

Correspondence: MT Brandl, Produce Safety and Microbiology Research Unit, Western Regional Research Center, Agricultural Research Service, U.S. Department of Agriculture, Albany, CA 94710, USA

E-mail: maria.brandl@ars.usda.gov

Received 24 March 2010; revised and accepted 24 June 2010; published online 5 August 2010 digestive vacuoles (phagosomes) of Acanthamoeba castellanii (Ly and Muller, 1990; Cirillo et al., 1997; Essig et al., 1997; Abu Kwaik et al., 1998) whereas Salmonella enterica serovars Dublin and Typhimurium multiply in Acanthamoeba rhysodes and Acanthamoeba polyphaga (Gaze et al., 2003; Tezcan-Merdol et al., 2004).

The resistance of certain intracellular pathogens to digestion by protozoa may coincidentally facilitate their ability to cause disease in their eukaryotic hosts. Phagocytosis in free-living protozoa shares basic mechanisms with that in human phagocytic cells, and conditions within the protozoan food vacuoles overlap with those in the macrophage phagosome (Lock et al., 1987; Jacobs et al., 2006; Cosson and Soldati, 2008). The interaction between pathogens and predatory amoebae has been implicated in the maintenance of bacterial virulence in various pathogens (Molmeret et al., 2005). For example, L. pneumophila and M. avium both show increased infectivity of human macrophages after passage through $A$. castellanii (Cirillo et al., 1994, 1997). On the basis of similarities in L. pneumophila mechanisms of infection of amoebae and mammalian 
cells, Molmeret et al. (2005) suggested that the intracellular lifestyle of human pathogens may have resulted from their adaptation to replication within free-living amoebae.

We have reported previously that $S$. Typhimurium can survive digestion by Tetrahymena at high rates and is released as viable cells in its fecal pellets in which it has enhanced survival compared with cells remaining undigested and free in suspension (Brandl et al., 2005). This is in contrast with L. monocytogenes, which is digested by the protist and detected infrequently in its fecal pellets (Brandl et al., 2005; Gourabathini et al., 2008). In addition, $S$. Typhimurium does not decrease the viability of Tetrahymena during its intravacuolar passage (Gourabathini et al., 2008). Therefore, its interaction appears to be different than with Acanthamoeba spp., which causes death of the protist (Gaze et al., 2003; Tezcan-Merdol et al., 2004; Feng et al., 2009). $S$. Typhimurium is an intracellular pathogen that has evolved specific mechanisms for its persistence and replication in eukaryotic cells. It is currently unclear whether the ability of the pathogen to resist digestion by Tetrahymena involves the same adaptations used to survive the phagocytic process of other eukaryotic cells.

In this study, we investigated the interaction of $S$. Typhimurium with Tetrahymena by microarray analysis of gene expression in $S$. Typhimurium cells residing in the Tetrahymena digestive vacuole. The global transcriptional response of this human pathogen to the Tetrahymena vacuolar environment indicates that it experiences conditions in the protozoan phagosome that overlap with those in macrophages and epithelial cells. In addition, cell viability assays showed that $S$. Typhimurium requires acid stress tolerance for survival to phagocytosis by Tetrahymena.

\section{Materials and methods}

Strains, plasmids and culture conditions

All strains and plasmids used in this study are listed in Table 1. Tetrahymena sp. MB125 was isolated from soil in California, belongs to an unknown species of this genus and has been described previously (Brandl et al., 2005). It was used in this study because of its release of numerous large fecal pellets containing viable $S$. enterica cells upon grazing on this pathogen (Figure 1).

S. enterica serovar Typhimurium SL1344 strains MB676 and MB681 are derivatives of SL1344 with a deletion in mgtC and adiAY, respectively. Mutants MB676 and MB681 were created using the lambda Red recombinase system (Datsenko and Wanner, 2000 ) to replace mgtC and adiAY, respectively, with the chloramphenicol acetyltransferase (CAT) cassette. All primers used for this procedure and others in this study are listed in Supplementary Table S1. The $m g t C$ and adiAY deletions were confirmed by PCR. Complemented mutant strains MB692 and MB694 were constructed by transforming MB676

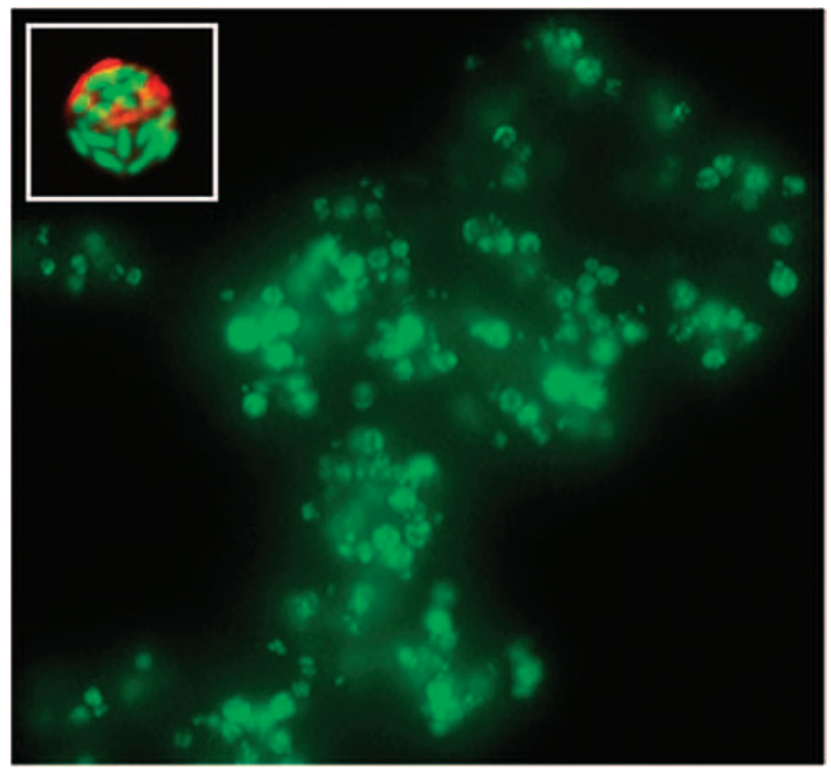

Figure 1 Epifluorescence micrograph of a large aggregate of fecal pellets released by Tetrahymena sp. upon feeding on $S$. Typhimurium and stained with SYTO 9 (Invitrogen). The inset shows a single optical scan through a Live/Dead BacLight-stained fecal pellet containing live (green) and dead (red) $S$. Typhimurium cells. The micrograph was captured with a Leica SP5 AOTF confocal microscope (Leica Microsystems, Wetzlar, Germany).

Table 1 Strains and plasmids used in this study

\begin{tabular}{lll}
\hline Strain name & Description & Reference \\
\hline MB125 & Tetrahymena spp. isolated from wet soil in California & Brandl et al., 2005 \\
MB282 & S. enterica serovar Typhimurium SL1344 & Hoiseth and Stocker, 1981 \\
MB676 & SL1344-derived; mgtC ORF replaced with CAT cassette & This study \\
MB681 & SL1344-derived; adiA and adiY ORFs replaced with CAT cassette & This study \\
MB692 & MB676-derived; complemented with pMTB687 & This study \\
MB694 & MB681-derived; complemented with pMTB688 & This study \\
Plasmid & & \\
pKD3 & Used in lambda Red-mediated recombination; contains CAT cassette & Datsenko and Wanner, 2000 \\
pKD46 & Lambda-Red recombinase expression plasmid & Datsenko and Wanner, 2000 \\
pBBR1MCS-5 & Broad-range cloning vector; gent ${ }^{\mathrm{R}}$ & Kovach et al., 1995 \\
pMTB687 & pBBR1MCS-5 ligated with mgtC ORF & This study \\
pMTB688 & pBBR1MCS-5 ligated with adiA-adiY ORFs & This study \\
\hline
\end{tabular}


and MB681 with the stably maintained plasmid pBBR1MCS-5 (Kovach et al., 1995) containing mgtC or adiAY, respectively. Briefly, mgtC or adiAY were PCR-cloned from strain SL1344 into pBBR1MCS-5 using primers $K p n I+m g t C \_F / P s t I+m g t C \_R$ or $K p n I+$ adiA_F/PstI + adiY_R. Plasmid pBBR1MCS-5 containing either mgtC (pMTB687) or adiAY (pMTB688) was electroporated into mutant MB676 or MB681, respectively. Transformants were isolated on LB agar containing gentamycin $\left(15 \mu \mathrm{g} \mathrm{ml}^{-1}\right)$ and X-gal. S. enterica SL1344 and derivatives were cultured in LB broth containing streptomycin $\left(30 \mu \mathrm{g} \mathrm{ml}^{-1}\right)$, and chloramphenicol $\left(20 \mu \mathrm{g} \mathrm{ml}^{-1}\right)$ and gentamycin $\left(15 \mu \mathrm{g} \mathrm{ml}^{-1}\right)$, as appropriate.

\section{Co-culture conditions}

Tetrahymena MB125 was grown in 2/3 strength Plate Count Broth (2/3 PCB) (Becton Dickinson, Franklin Lakes, NJ, USA) for 2 days at $28^{\circ} \mathrm{C}$, with agitation at 50 r.p.m. It was then centrifuged at $200 \times g$ for $2 \mathrm{~min}$ and repeatedly washed with half volumes of sterile deionized $\mathrm{H}_{2} \mathrm{O}\left(\mathrm{dH}_{2} \mathrm{O}\right)$, incubating the cells for 15 min during each wash to minimize lysis by osmotic shock followed by centrifugation. The cell concentration was measured with a hemacytometer and was adjusted to $1 \times 10^{5} \mathrm{cells} \mathrm{ml}^{-1}$. SL1344 cells were cultured to the mid-log phase of growth in LB broth at $28{ }^{\circ} \mathrm{C}$ and washed twice in sterile $\mathrm{dH}_{2} \mathrm{O}$. The $\mathrm{OD}_{600}$ of the suspension was adjusted to 0.2 and the suspension was combined with that of washed Tetrahymena cells at a bacteria:ciliate ratio of 1000:1 in sterile $\mathrm{dH}_{2} \mathrm{O}$. The mixed suspensions were incubated at $28^{\circ} \mathrm{C}$ and 50 r.p.m. for $3 \mathrm{~h}$ until most bacteria were ingested by Tetrahymena. This was determined by staining the suspension with SYTO 9 (Invitrogen, Carlsbad, CA, USA) $\left(10 \mu \mathrm{M}\right.$ final) for $30 \mathrm{~min}$ at $23^{\circ} \mathrm{C}$ in the dark and visualized by epifluorescence microscopy with a Leica DMR microscope.

\section{RNA extraction}

Eight co-cultures of $50 \mathrm{ml}$ were centrifuged at $800 \times g$ for $2 \mathrm{~min}$. The resulting pellet was enriched for Tetrahymena cells containing ingested SL1344 cells as most uningested free bacteria were not pelleted and remained in suspension. The supernatant was quickly removed and the pellet was resuspended in ice-cold lysis buffer (0.5\% SDS, 19\% ethanol and 1\% phenol in $\mathrm{H}_{2} \mathrm{O}$ ), on the basis of the protocol by Eriksson et al. (2003). The suspension containing lysed Tetrahymena cells was centrifuged at $3200 \times g$ for $5 \mathrm{~min}$ at $4{ }^{\circ} \mathrm{C}$. The bacterial pellet was stored at $-80^{\circ} \mathrm{C}$. RNA extraction was performed with the Promega SV Total RNA Isolation kit per the manufacturer's specifications, except that bacterial pellets were first treated with $50 \mu \mathrm{g} \mathrm{ml}^{-1}$ of lysozyme (Fisher Scientific, Pittsburgh, PA, USA). RNA from two different sources was used as control. The first control RNA was isolated from $50 \mathrm{ml}$ of SL1344 cells incubated in sterile $\mathrm{dH}_{2} \mathrm{O}$ $\left(\mathrm{OD}_{600}=0.2\right)$ for $3 \mathrm{~h}$, similar to the SL1344 cells mixed with Tetrahymena. The second control RNA was isolated from $20 \mathrm{ml}$ of SL1344 cells grown to the mid-log phase of growth in LB broth. Both suspensions were centrifuged at $3200 \times g$ for $5 \mathrm{~min}$ at $4{ }^{\circ} \mathrm{C}$, followed by addition of lysis buffer, centrifugation and RNA extraction, as described above. Three biological replicates were used for each type of suspension. RNA integrity was assessed with the Agilent 2100 Bioanalyzer and only RNA of high quality was used for microarray hybridizations.

\section{Microarray analysis}

Custom, whole-genome $S$. enterica LT2 arrays were prepared at the Western Regional Research Center. Each array contained 4360 PCR-generated open-reading frames (including 104 from virulence plasmid pSLT) from $S$. enterica serovar Typhimurium strain LT2 printed onto Ultra-GAPS glass slides (Corning, Corning, NY, USA) with a Gene Machine Omnigrid Accent Arrayer (Genomic Solutions, Ann Arbor, MI, USA). Labeled nucleotides were prepared and hybridized to arrays as described previously (Kyle et al., 2010) on the basis of the method developed by Eriksson et al. (2003), with a few modifications. Briefly, $20 \mu \mathrm{g}$ of RNA from co-cultures and controls was reverse-transcribed and Cy3-dCTP (GE Healthcare, Waukesha, WI, USA) incorporated into cDNA using the Fairplay III Microarray Labeling Kit (Stratagene, La Jolla, CA, USA). A 2- $\mu$ g volume of SL1344 genomic DNA was labeled with Cy5-dCTP (GE Healthcare) using Klenow (New England Biolabs, Ipswich, MA, USA) and served as the reference signal on the arrays. Labeling efficiency was determined with a Nanodrop 2000 Spectrophotometer (Thermo Scientific, Waltham, MA, USA). All cDNA solutions were adjusted to the same label concentration in the Pronto! cDNA hybridization solution (Corning) and were hybridized to the arrays overnight at $42{ }^{\circ} \mathrm{C}$ according to the manufacturer's protocol. For each experimental condition, cDNA was prepared from three replicate suspensions (biological replicates) and each cDNA was hybridized to three replicate arrays (technical replicates) at random among several slides. The slides were washed and then scanned with an Axon Genepix 4000b scanner and the Axon Genepix Pro 4.1 software (Molecular Devices, Sunnyvale, CA, USA).

To address spatial dye effects or any disparity in the amounts of spotted cDNA, data were normalized by setting the median log (Cy3/Cy5 signals) of all of the spots on the same array to zero. Biological replicates were then analyzed by analysis of variance $(P<0.05)$ with the Genespring version 7 software (Agilent Technologies, Santa Clara, CA, USA). From these data sets, only genes that were differentially regulated at least two-fold and for which the mean change in expression passed the Benjamini-Hochberg False Discovery Rate test $(P<0.05)$ were included in gene lists. Gene lists were generated for comparison of expression in SL1344 cells in Tetrahymena phagosomes with that in (1) $\mathrm{H}_{2} \mathrm{O}$ and (2) LB broth, and for comparison of expression in $\mathrm{H}_{2} \mathrm{O}$ versus that 
in LB broth. All final gene lists are presented as Supplementary Table S2. Genes of particular interest were those that were differentially regulated in $S$. Typhimurium in Tetrahymena vacuoles versus both in $\mathrm{H}_{2} \mathrm{O}$ and in $\mathrm{LB}$ broth.

Determination of cell viability and population density in fecal pellets

After co-incubation of SL1344 and Tetrahymena MB125 as described above, the suspension was filtered through Millipore black Isopore membranes $(0.2 \mu \mathrm{m}$ pore size, $25 \mathrm{~mm}$ diameter) and cell viability assessed as described previously (Brandl et al., 2005). Briefly, filters were gently submerged in $\mathrm{H}_{2} \mathrm{O}$ to allow Tetrahymena cells to swim away from the filter leaving behind free bacteria and pellets. The filters were removed from the water and drained. For assessment of bacterial acid resistance, the filters were submerged again in $\mathrm{H}_{2} \mathrm{O}$ at $\mathrm{pH} 3.4$ for $1 \mathrm{~h}$. The filters were rinsed by submersion in $\mathrm{H}_{2} \mathrm{O}$ five times and drained.

Viability staining of cells on the filters was performed with Live/Dead BacLight (Invitrogen) by placing the filter into the stain solution $(1.5 \mu \mathrm{l}$ of each dye per $\mathrm{ml}$ ) for $25 \mathrm{~min}$ at $24{ }^{\circ} \mathrm{C}$ in the dark. The filters were rinsed by submersion in $\mathrm{H}_{2} \mathrm{O}$ and cells were viewed under an epifluorescence microscope with a fluorescein filter. The ratio of dead cells (red fluorescence) to viable cells (green fluorescence) was estimated in at least 50 fecal pellets in each of three replicate co-cultures. This ratio was estimated also for 10-30 cells external to each fecal pellet to assess the proportion of viable cells among cells remaining uningested and free in the mixed suspension. Figure 1 shows pellets produced by Tetrahymena and the presence of live and dead $S$. Typhimurium cells in the pellets, as shown by Live/Dead BacLight stain.

The viability of the $\triangle a d i A Y$ and $\triangle m g t C$ mutants, of the complemented mutants and of the wild-type SL1344 in fecal pellets was assessed as described above. Cell density in pellets was estimated for the $\Delta m g t C$ mutant, the complemented mutant and the wild-type strain by counting the total cell number per pellet for at least 50 pellets. For strain comparisons, an aliquot of the bacterial inoculum was stained with Live/Dead BacLight to ensure that viability did not differ among strains before preparation of the cocultures with Tetrahymena. Cell viability and density data were analyzed statistically with a two-tailed $t$-test or a one-way analysis of variance followed by Tukey's multiple comparison test, with $P<0.05$. All tests were performed with the Prism software version 5.02 (GraphPad Software, La Jolla, CA, USA). Experiments were performed at least twice.

\section{Results}

Transcriptional profile of S. Typhimurium in

Tetrahymena phagosomes

The gene expression profile of $S$. Typhimurium SL1344 residing in Tetrahymena food vacuoles was compared with that of cells incubated in $\mathrm{H}_{2} \mathrm{O}$ or in LB broth. Because $S$. Typhimurium cells were harvested from the food vacuoles $3 \mathrm{~h}$ after the start of co-incubation of the two microorganisms in water, it is likely that a considerable percentage of pathogen cells spent a significant amount of time in water before falling prey to the protist. Therefore, we considered incubation of $S$. Typhimurium in water without the protist as the most appropriate control to identify genes differentially regulated in Tetrahymena phagosomes. However, because microarray studies of the $S$. Typhimurium transcriptome in other eukaryotic cells used LB broth as a control (Eriksson et al., 2003; Hautefort et al, 2008), we included LB broth as an additional control to compare our results with previously published data. Our microarray analysis showed that expression of 989 and 1282 genes changed at least two-fold in the Tetrahymena phagosome compared with in water and in LB medium, respectively (Supplementary Table S2). Overall, more genes were downregulated (520 and 811) than upregulated (469 and 471) in the Tetrahymena phagosome versus in $\mathrm{H}_{2} \mathrm{O}$ and in $\mathrm{LB}$ broth, respectively (Figure 2 and Supplementary Table S2). Notable exceptions, as illustrated by Categories of Orthologous Genes (COG) categories with $\mathrm{H}_{2} \mathrm{O}$ as the control environment, include upregulated genes involved in energy production and conversion, nucleotide transport and metabolism, translation, cell motility and intracellular trafficking and secretion (Figure 2). Also of interest is the higher number of upregulated than downregulated genes that have a role in replication, recombination and repair, and in cell wall/ membrane biogenesis. A close examination of the detailed gene lists showed that phagosome conditions induced numerous genes involved in anerobiosis. These included the hydrogenase operons hyc, hyd and hyp, and the reductase genes $d m s A B$ and frdBA (Table 2).

We also observed increased expression of various virulence genes, several of which are associated with the Salmonella-containing vacuole of the macrophage (Eriksson et al., 2003; Faucher et al., 2006). These included the PhoP/PhoQ-regulated genes pagC, pagK, envE, virK, mgtB and mgtC (Table 2). Additional Salmonella-containing vacuole-associated virulence genes upregulated in the Tetrahymena phagosome were those encoding the Salmonella Pathogenicity Island-2 (SPI-2) typeIII secretion system apparatus protein $\mathrm{SsaV}$ and the secreted effector proteins SifB and SopB (Faucher et al., 2006) (Table 2).

Other genes with increased expression in Tetrahymena have a role in acid stress-response (adiAY) and in antibiotic or antimicrobial resistance (mar$R A B$, emrA and $y d d G$ ) (Table 2). $y d d G$ codes for a porin involved in the efflux of methyl viologen, which generates oxygen radicals (Santiviago et al., 2002). In addition, a small number of upregulated genes are involved in osmotic stress, namely $k d p A$ 


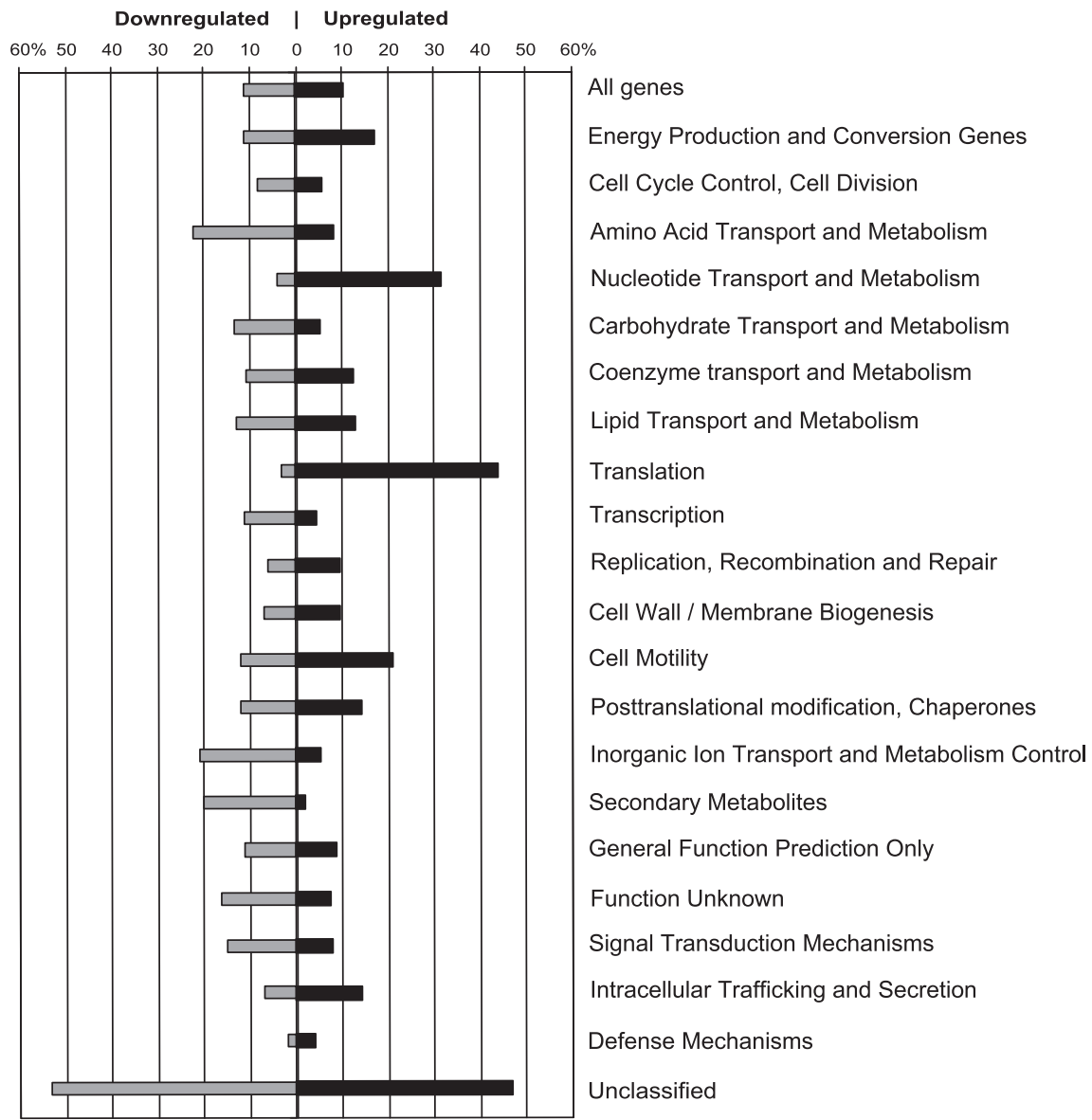

Figure 2 Differential expression of genes within Categories of Orthologous Genes (COG) in S. Typhimurium cells residing in Tetrahymena vacuoles compared with that in $\mathrm{H}_{2} \mathrm{O}$, as shown by microarray analysis. The bars represent the percentage of genes with a change in transcription of at least two-fold within a given category.

and $k p d B$, and ompW. The $k d p$ operon is induced upon osmotic upshift through loss of turgor (Balaji et al., 2005), whereas ompW encodes a porin involved in osmoregulation. However, there is also evidence that similar to YddG, OmpW exports methyl viologen and may work in conjunction with YddG (Gil et al., 2007).

When examining expression changes in S. Typhimurium in Tetrahymena phagosomes with LB broth as a control environment, we identified additional genes that are upregulated also in macrophages and epithelial cells. Supplementary Table S3 lists a subset of 146 and 92 genes that are upregulated in Tetrahymena versus in LB and which were reported previously to also increase in expression after $8 \mathrm{~h}$ in J774-A.1 macrophages and after $2 \mathrm{~h}$ in HeLa cells, respectively (Supplementary Table S1 in reference Hautefort et al., 2008). Commonalities in differentially regulated genes in the latter cells and in Tetrahymena were observed at other incubation times as well, but the number of overlapping upregulated genes was smaller. Of the genes that showed increased expression in both Tetrahymena and macrophages, 53\% were upregulated also in HeLa cells (Supplementary Table S3). Common
Tetrahymena- and macrophage-upregulated genes in comparison to LB included, but were not limited to, the following categories: SPI-2 (ssr $A, s s a B / G / H / I$ / $L / V / R$ and $s s c A$ ); oxidative stress (ycfR, trx $C$ and $i b p B$-in addition to $d p s$, yfiA and katG, which are involved in oxidative stress response also but were not upregulated in macrophages); osmotic stress (osmB); SOS response (uvrB and umuC); multidrug resistance (emrD); phosphate starvation ( $p s i F) ; \mathrm{Mg}^{2+}$ transport (mgtB); anerobic metabolism (hydN, hycA and $f h l A$ ) and 49 hypothetical proteins (Supplementary Table S3). It is noteworthy that as observed in macrophages, the iron acquisition genes ent $A B C E$ were highly downregulated in Tetrahymena. Along with downregulation of sit $A B C D$, this suggests the presence of iron and manganese in the protozoan phagosome.

Passage through Tetrahymena induces an acid stress response

To determine whether the acidic conditions in Tetrahymena digestive vacuoles induce an adaptive tolerance to low $\mathrm{pH}$ in $S$. Typhimurium, we measured the viability of strain SL1344 cells in 
Table 2 Select categories of $S$. Typhimurium genes upregulated at least two-fold in the Tetrahymena phagosome compared with that in water and in LB broth, as determined by microarray analysis

\begin{tabular}{|c|c|c|c|c|}
\hline \multirow[t]{2}{*}{ Locus } & \multirow[t]{2}{*}{ Name } & \multicolumn{2}{|c|}{ Fold change } & \multirow[t]{2}{*}{ Function } \\
\hline & & vs $\mathrm{H}_{2} \mathrm{O}$ & vs $L B$ & \\
\hline \multicolumn{5}{|c|}{ Anaerobic energy generation/alternate electron acceptors } \\
\hline STM0964 & $d m s A$ & 17.87 & 14.40 & Anaerobic DMSO reductase subunit-A \\
\hline STM0965 & $d m s B$ & 11.79 & 18.94 & Anaerobic DMSO reductase subunit-B \\
\hline STM1538 & hydA & 2.36 & 4.31 & Putative Ni-Fe hydrogenase-1 large subunit \\
\hline STM1539 & hydB & 3.16 & 6.22 & Putative Ni-Fe hydrogenase-1 small subunit \\
\hline STM2063 & phsC & 2.33 & 3.50 & $\mathrm{H}_{2} \mathrm{~S}$ production from thiosulfate \\
\hline STM2065 & phs $A$ & 4.78 & 5.46 & $\mathrm{H}_{2} \mathrm{~S}$ production from thiosulfate \\
\hline STM2529 & & 7.27 & 7.14 & Putative anaerobic DMSO (dimethylsulfoxide) reductase \\
\hline STM2530 & & 8.69 & 8.51 & Putative anaerobic DMSO reductase \\
\hline STM2843 & hydN & 5.30 & 6.85 & Formate dehydrogenase-H, [4Fe-4S] ferredoxin subunit \\
\hline STM2845 & hycI & 5.07 & 5.80 & Protease involved in processing the C-terminal end of $\mathrm{HycE}$ \\
\hline STM2846 & hycH & 6.62 & 5.59 & Processing of HycE (part of the formate-hydrogen-lyase (FHL) complex) \\
\hline STM2848 & hycF & 5.66 & 7.87 & Hydrogenase-3, putative quinone oxidoreductase \\
\hline STM2850 & hycD & 3.42 & 5.64 & Hydrogenase-3, membrane subunit (part of FHL complex) \\
\hline STM2852 & hycB & 4.86 & 7.62 & Hydrogenase-3, Fe-S subunit (part of FHL complex) \\
\hline STM2853 & hycA & 3.78 & 6.13 & Transcriptional repressor of hyc and hyp operons \\
\hline STM2854 & hурA & 5.57 & & Functions as nickel donor for HycE of hydrogenlyase-3 in FHL complex \\
\hline STM2855 & hypB & 8.84 & 2.79 & Hydrogenase- 3 accessory protein, assembly of metallocenter \\
\hline STM2856 & hypC & 3.93 & 2.80 & Putative hydrogenase expression/formation protein \\
\hline STM2857 & hypD & 8.62 & 3.09 & Putative hydrogenase expression/formation protein \\
\hline STM2858 & hypE & 3.77 & 2.98 & Putative hydrogenase expression/formation protein \\
\hline STM2859 & fhlA & 2.69 & 2.55 & Formate hydrogenlyase transcriptional activator for $f d h F, h y c$ and hyp operons \\
\hline STM3143 & hybG & 2.20 & 2.25 & Hydrogenase- 2 operon protein \\
\hline STM3144 & hybF & 3.81 & 2.84 & Putative hydrogenase-2 expression/formation protein \\
\hline STM3145 & hybE & 3.28 & 2.64 & Hydrogenase-2 operon protein \\
\hline STM3146 & hybD & 4.70 & 3.52 & Putative processing element for hydrogenase-2 \\
\hline STM3147 & hybC & 4.64 & 2.95 & Hydrogenase-2, large subunit \\
\hline STM3148 & $h y b B$ & 8.96 & 5.20 & Putative cytochrome Ni/Fe component of hydrogenase-2 \\
\hline STM3149 & hybA & 9.29 & 5.92 & Putative hydrogenase operon protein \\
\hline STM3150 & hypO & 11.29 & 4.61 & Hydrogenase-2, small subunit \\
\hline STM4285 & $f d h F$ & 17.52 & 14.14 & Formate dehydrogenase \\
\hline STM4342 & $f r d B$ & 2.54 & 3.18 & Fumarate reductase, Fe-S subunit \\
\hline STM4343 & frdA & 3.20 & & Fumarate reductase, flavoprotein subunit \\
\hline
\end{tabular}

Virulence and antimicrobial resistance genes

Virulence plasmid

$\begin{array}{llll}\text { pSLT012 } & \text { orf7 } & 4.07 & 3.4 \\ \text { pSLT013 } & \text { pefI } & 5.25 & 6.9 \\ \text { pSTL096 } & \text { trbE } & 2.29 & 2.16\end{array}$

PhoP-PhoQ-activated genes STM0628 pagP 3.56

STM1242 envE $\quad 2.41$

STM1254

STM1867 pagK 2.23

STM2781 virK $\quad 6.67$

STM2782 mig-14 $\quad 6.94$

STM3763 mgtB 3.32

STM3764 mgtC 81.98

Other virulence genes

$\begin{array}{llll}\text { STM1091 } & \text { sopB } & 2.02 & \\ \text { STM1414 } & \text { ssaV } & 3.87 & 5.66 \\ \text { STM1602 } & \text { sifB } & 2.84 & 3.08\end{array}$

Antimicrobial resistance

STM1518 marB 2.33

$\begin{array}{lll}\text { STM1519 } \quad \text { marA } & 5.76\end{array}$

STM1520 marR $\quad 8.58$

STM1571 $\quad y d d G \quad 4.17$

STM2814 emrA $\quad 5.06$
3.43

2.16

Putative bacterial regulatory protein, luxR family

Transcriptional regulator of pef operon

Conjugative transfer

Lipid-A palmitoyl transferase required for resistance to antimicrobial peptides

Putative envelope protein

2.16 Putative envelope protein required for survival in macrophages

5.51 Putative outer membrane lipoprotein

2.47 Putative virulence protein

Putative virulence protein

Required for virulence and resistance to antimicrobial peptides

4.46 $\mathrm{Mg}^{2+}$-transporting ATPase

73.67 Putative ion homeostasis protein required for persistence in macrophages

Inositol polyphosphatase required for entry into intestinal epithelial cells

Type-III secretion system apparatus protein

Secreted effector

Multiple antibiotic resistance protein

Transcriptional activator of defense systems, multiple antibiotic

resistance protein

Transcriptional repressor of mar operon, multiple antibiotic

resistance protein

Required for efflux of methyl viologen, a quaternary ammonium compound

Multidrug resistance protein 
Table 2 (Continued)

\begin{tabular}{|c|c|c|c|c|}
\hline \multirow[t]{2}{*}{ Locus } & \multirow[t]{2}{*}{ Name } & \multicolumn{2}{|c|}{ Fold change } & \multirow[t]{2}{*}{ Function } \\
\hline & & vs $\mathrm{H}_{2} \mathrm{O}$ & vs $L B$ & \\
\hline \multicolumn{5}{|c|}{ Osmotic stress } \\
\hline STM0705 & $k d p B$ & 5.15 & 9.31 & P-type ATPase, high-affinity potassium transport system, B-chain \\
\hline STM0706 & $k d p A$ & 9.01 & 11.67 & P-type ATPase, high-affinity potassium transport system, A-chain \\
\hline STM1732 & ompW & 9.99 & & Porin involved in osmoregulation \\
\hline \multicolumn{5}{|l|}{ Acid stress } \\
\hline STM4295 & $\operatorname{adi} Y$ & 4.12 & 6.81 & Transcriptional activator of adiA (AraC/XylS family) \\
\hline STM4296 & adiA & 6.20 & 5.71 & Arginine decarboxylase, catabolic; inducible by acid \\
\hline
\end{tabular}

fecal pellets released by the protist and subsequently exposed to acidic conditions. The mean proportion of viable cells in pellets after acid treatment at $\mathrm{pH} 3.4$ was $67.7 \%$ with a standard deviation (s.d.) of 1.47, and was significantly greater than that among cells remaining uningested and free in suspension (7.7\%; s.d., 0.95) ( $t$-test, $P<0.0001)$. The frequency distribution of percentage viability among the two cell types clearly illustrates a shift toward greater viability of SL1344 in the pellets as compared with that of free cells upon exposure of both to acid stress (Figure 3). Although the percentage of viable cells in individual pellets was variable after exposure to $\mathrm{pH} 3.4$, with the exception of one outlier pellet, it did not reduce below 31\% (Figure 3a). By contrast, the percentage viability among free cells after acid treatment was lower than $31 \%$ in $90 \%$ of the areas sampled on the filter (Figure 3b).

Arginine-dependent acid resistance in S. Typhimurium within Tetrahymena phagosomes

The viability of the SL1344 $\Delta$ adiAY mutant (MB681) and of its complemented strain (MB694) was compared to that of the wild-type strain in Tetrahymena fecal pellets. After a 3-h incubation of these strains with Tetrahymena, MB681 showed a mean percent viability in pellets of $66.03 \%$ (s.d., 3.53), versus $84.82 \%$ (s.d., 2.49) and $95.50 \%$ (s.d., 1.49) for MB694 and the wild-type strain, respectively. Although complete mortality of MB681 was not observed, MB681 had lower survival in the Tetrahymena phagosome than MB694 and the wild-type strain (Tukey's multiple comparison test, $P<0.05$ ). The percentage viable cells among free cells uningested by Tetrahymena did not differ significantly between the three strains (analysis of variance, $P=0.59$ ). The distribution of percentage viable MB681 cells in pellets was broad, ranging from $100 \%$ mortality in one pellet to over $96 \%$ viable cells in others (Figure 4a). By contrast, the proportion of viable cells of the complemented mutant (MB694) in the pellets did not reduce below 46\% (with the exception of one outlier), and in half of the
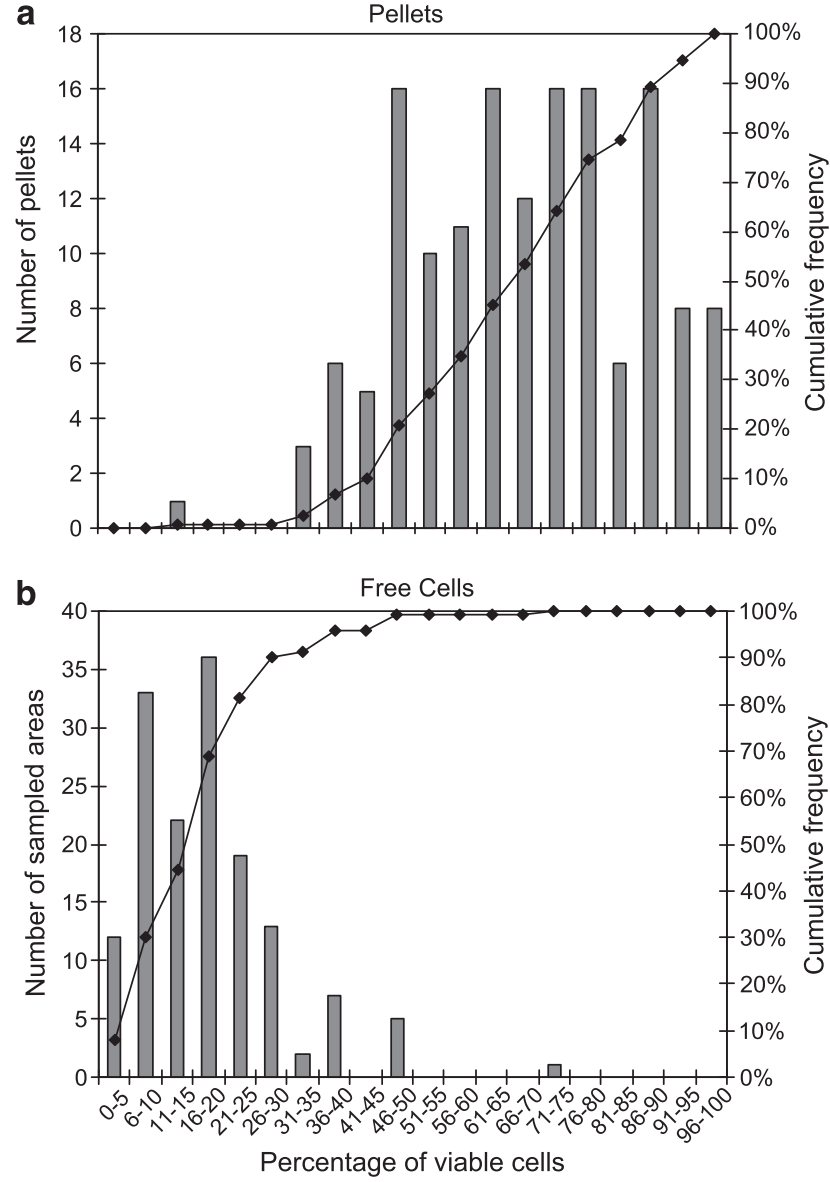

Figure 3 Frequency distribution of percent viable $S$. Typhimurium cells in individual Tetrahymena fecal pellets and in sampled areas of free uningested cells after exposure to $\mathrm{pH}$ 3.4. Cell viability was assessed with the Live/Dead BacLight stain. The bars represent the number of pellets (a) and the number of sampled areas (for free cells) (b), with a proportion of viable $S$. Typhimurium cells in a given range. The data on the solid line, plotted against the right $y$-axis, represent the cumulative frequency of observations in each distribution.

pellets the percentage of viable cells was greater than $85 \%$ (Figure $4 \mathrm{~b}$ ). The wild-type strain had the highest viability of all three strains, with $76 \%$ of the pellets containing at least $96 \%$ of viable cells (Figure 4c). 

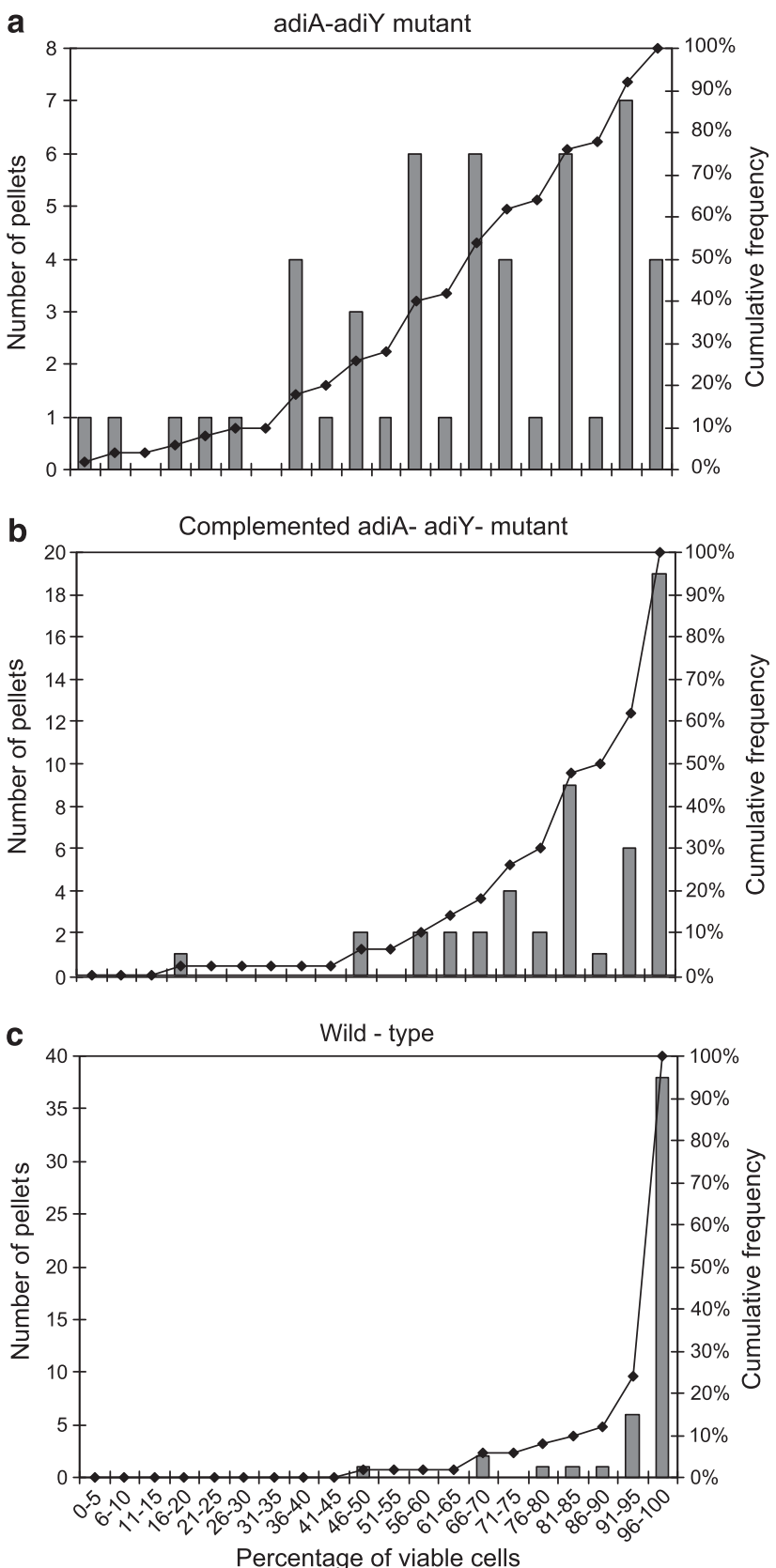

Figure 4 Frequency distribution of percent viable $S$. Typhimurium cells in individual fecal pellets released by Tetrahymena during co-culture of the two microorganisms, as assessed with the Live/Dead BacLight stain. The number of individual pellets with a proportion of viable $S$. Typhimurium cells in a given range (bars) and the cumulative frequency of pellets across the range of percent viable cells (solid line, right $y$-axis) are illustrated for the S. Typhimurium $\triangle$ adiA $\Delta a d i Y$ mutant (MB681) (a), the complemented mutant (MB694) (b) and the wild-type strain (c).

Role of MgtC in S. Typhimurium cell density in Tetrahymena fecal pellets

The macrophage virulence factor MgtC was investigated for its potential role in the digestion resistance of the pathogen. Whereas mgtB encodes an $\mathrm{Mg}^{2+}$ transporter, $m g t C$ is involved in regulating membrane potential (Gunzel et al. 2006). With an increase in expression of 82- and 74-fold compared with that in water and LB broth, respectively, mgtC was one of the most highly induced genes in Tetrahymena (Table 2). The cell density and viability of the $\Delta m g t C$ mutant (MB676), its complemented strain (MB692) and the wild-type strain of SL1344 were compared in the fecal pellets produced by co-culture of each strain with Tetrahymena. Live/ Dead staining of the cells in the fecal pellets showed that their viability did not differ between each strain (data not shown). However, the mean cell density per pellet for the mutant MB676 (24.3; s.d., 1.5) was significantly greater than that of the wild-type (17.8; s.d., 0.7) and the complemented mutant MB 692 (17.3; s.d., 0.7) (Tukey's multiple comparison test, $P<0.05)$. Compared with the wild-type and the complemented mutant, the frequency distribution of the number of mutant MB676 cells in the pellets was shifted toward a higher cell density (Figure 5).

\section{Discussion}

As intracellular pathogens have evolved to resist or escape phagosomal conditions, grazing protozoa may represent an environmental reservoir for these pathogens. Understanding the interaction between pathogenic bacteria and protozoan grazers may further our understanding of the factors that allow persistence of pathogens in the environment. We have reported previously that the intracellular pathogen, S. enterica, can remain undigested in Tetrahymena and that most cells remain viable upon their release in its fecal pellets (Brandl et al., 2005). The viability of cells within nascent fecal pellets is thus a direct result of survival within the Tetrahymena phagosome.

Bacteria that resist degradation in Tetrahymena phagosomes must counter harsh conditions such as acidification from proton-translocating ATPases, oxidative stress caused by reactive oxygen species, the presence of hydrolytic enzymes and reduced oxygen tension (Fok and Allen, 1975; Jacobs et al., 2006). Our global transcriptional analysis of S. Typhimurium SL1344 cells in Tetrahymena phagosomes showed extensive upregulation of the hyc, hyp, hyd and hyb hydrogenase operons, which function in anerobiosis (Vignais and Colbeau, 2004), thus, indicating a metabolic shift to an anaerobic lifestyle. Hyc and Hyp are involved in fermentative $\mathrm{H}_{2}$ evolution as part of the formate $\mathrm{H}_{2}$ lyase (FHL) complex, whereas Hyd and Hyb are linked to respiratory fumarate reduction (Richard et al., 1999; Zbell et al., 2007; Zbell and Maier, 2009). Deletion of hyd and hyb in $S$. Typhimurium results in colonization deficiency in a mouse model (Maier et al., 2004). Upregulation of the terminal reductases for fumurate and dimethylsulfoxide in the phagosome suggests that oxygen is highly limiting and alternate terminal electron acceptors may be preferred by $S$. Typhimurium. Overall, this 
270
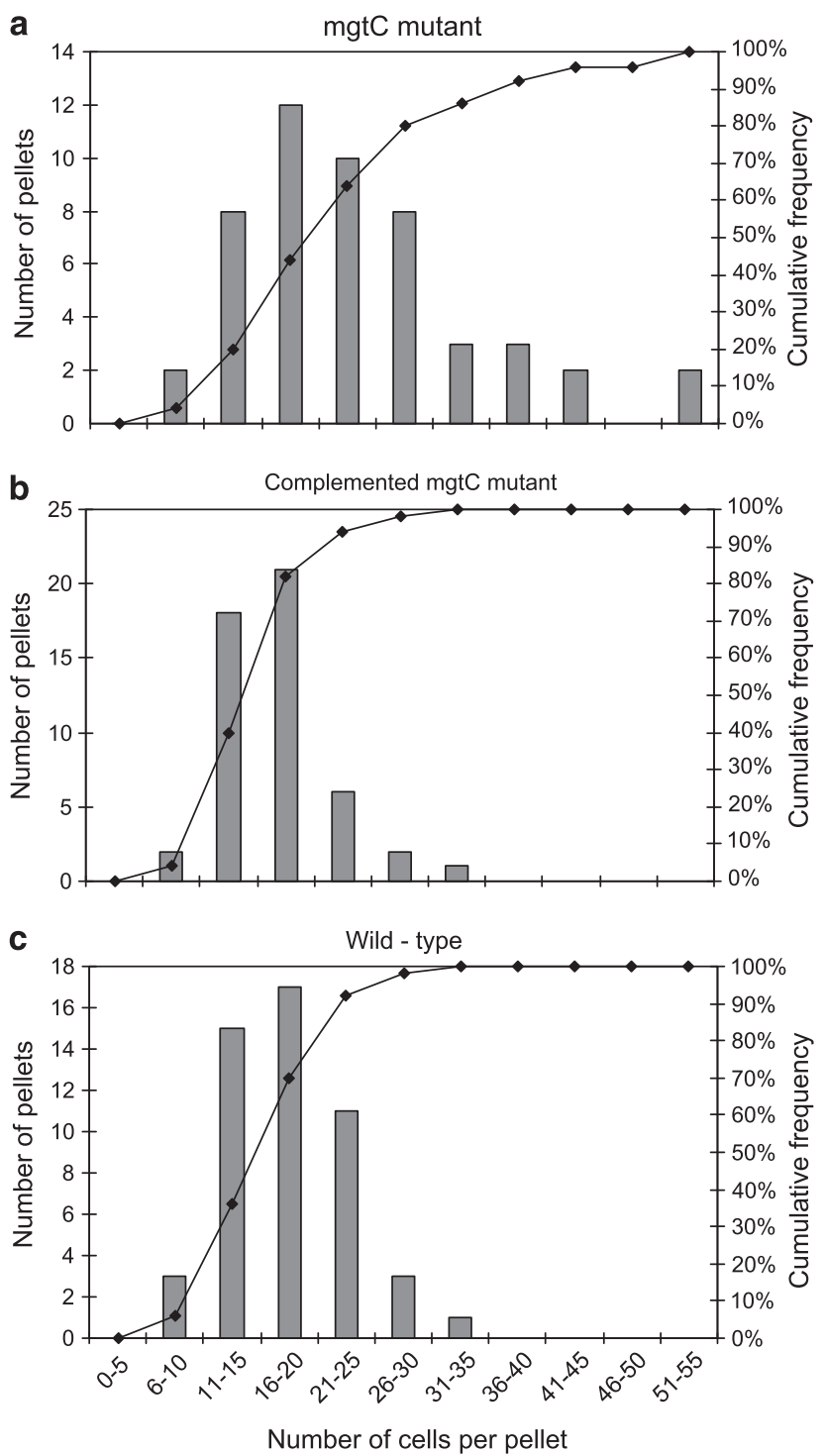

Figure 5 Frequency distribution of the number of $S$. Typhimurium cells in individual fecal pellets released by Tetrahymena during co-culture of the two microorganisms, as assessed with the Live/Dead BacLight stain. The number of individual pellets with an $S$. Typhimurium cell density in a given range (bars) and the cumulative frequency of pellets across the range of cell density (solid line, right $y$-axis) are illustrated for the $S$. Typhimurium $\Delta m g t C$ mutant (MB676) (a), the complemented mutant (MB692) (b) and the wild-type strain (c).

metabolic adaptation, along with increased translation and expression of genes that function in cell replication and wall/membrane biogenesis, may be indicative of the ability of $S$. Typhimurium to grow in the Tetrahymena phagosome. Further evidence of such a phenomenon still needs to be obtained.

Several $S$. Typhimurium genes that are upregulated in Tetrahymena phagosomes as compared with that in $\mathrm{H}_{2} \mathrm{O}$ are also induced in macrophages. These included the antimicrobial resistance genes marR $A B$ and emrA, of which increased expression was observed in the macrophage Salmonella-containing vacuole (Eriksson et al., 2003). Also noteworthy is the higher transcription of several virulence genes in the Tetrahymena phagosomes, including those of the PhoP-PhoQ regulon ( $p a g P / C / K$, envE, virK, mig-14 and mgtBC) and others belonging to SPI-1 (sopB) and SPI-2 (ssaV, sifB). The PhoP-PhoQ system is required for survival of $S$. Typhimurium and expression of SPI-2 genes within macrophages (Miller et al., 1989; Fass and Groisman, 2009). PagP is involved in remodeling of the lipid-A domain of lipopolysaccharide (Bishop, 2005), whereas Mig-14 and VirK promote resistance to antimicrobial peptides produced in macrophages (Brodsky et al., 2005). Low amounts of $\mathrm{Ca}^{2+}$ and $\mathrm{Mg}^{2+}$ (Groisman, 2001), acidic pH (Prost et al., 2007) and antimicrobial peptides (Bader et al., 2005) are the environmental cues for PhoPQ-mediated regulation. Thus, upregulation of $\operatorname{mgt} B$, adiAY and a variety of genes involved in antimicrobial resistance correlates well with the activation of the PhoPQ regulon in the Tetrahymena phagosome.

Using LB culture medium as a common control environment, we compared the microarray data obtained in this study with that reported by Hautefort et al. (2008) regarding $S$. Typhimurium gene expression in macrophages and epithelial cells. This comparative analysis provided evidence that the pathogen experiences physicochemical conditions in the Tetrahymena phagosome that overlap with those encountered in macrophages and epithelial cells. Commonalities between the protist vacuoles and vacuoles of at least one of the two other cell types on the basis of transcriptional profiles include conditions of acid, oxidative and osmotic stress, low magnesium and phosphate concentrations, presence of antimicrobials and conditions inducing the SOS response and SPI-2. This overall response of $S$. Typhimurium to a variety of stresses in intravacuolar environments may underlie its ability to resist protozoan digestion. Of particular interest are the 49 hypothetical proteins that are part of the transcriptional signature of the pathogen in both the protist and macrophages, and which may represent proteins with unknown function that are crucial to the survival of $S$. Typhimurium in phagocytic cells.

Despite this overlap in transcriptional profile, the considerably larger sets of $S$. Typhimurium genes upregulated in macrophages and HeLa cells (Hautefort et al., 2008) indicate that significant differences also exist. S. enterica does not have any detectable cytotoxic effect in Tetrahymena (Brandl et al., 2005; Gourabathini et al., 2008), in contrast to Acanthamoeba spp., which are killed by $S$. enterica and other pathogenic species (Abu Kwaik et al., 1998; Gaze et al., 2003; Tezcan-Merdol et al., 2004; Matz et al., 2008; Feng et al., 2009). Hence, the role of SPI-2 and other virulence determinants in its resistance to digestion by Tetrahymena is less clear than their potential pathogenic function during interaction with Acanthamoeba rhysodes, in which SPI genes are also 
induced (Feng et al., 2009). The possibility remains that increased expression of virulence genes in $S$. Typhimurium in Tetrahymena is simply a response to environmental signals present also in host phagocytic cells, particularly low $\mathrm{pH}$ (Yu et al., 2010). Further investigation of the response of Tetrahymena to this enteric pathogen may provide more insight into their interaction.

$m g t C$ is one of the most highly upregulated $S$. Typhimurium genes in macrophages (Eriksson et al., 2003) and in HeLa cells (Hautefort et al., 2008), and is required for its long-term phagosomal survival (Alix and Blanc-Potard, 2007). Similarly, $m g t C$ had the highest differential expression in our study, yet the $\Delta m g t C$ mutant was as viable as the wild-type strain in Tetrahymena pellets, suggesting that it was not impaired for survival in its phagosome. Possibly, the passage time of the pathogen before release at the cytoproct, which we estimated to be approximately $1 \mathrm{~h}$ (Brandl et al., 2005), is not sufficiently long for this mutation to affect cell survival in Tetrahymena. Paradoxically, the $\Delta m g t C$ mutant had a greater cell density than the wild type in the fecal pellets. Complementation of the mutant caused lower cell density in the pellets, thus supporting a role for MgtC in this phenotype. Because growth in low- $\mathrm{Mg}^{2+}$ medium causes cell elongation and aggregation of MgtC-minus mutants (Rang et al., 2007), Tetrahymena may have ingested cell aggregates during feeding on this mutant in $\mathrm{H}_{2} \mathrm{O}$, leading to a greater cell density in its food vacuoles. However, it is unclear if Tetrahymena would be able to feed on such aggregates because of their size.

In light of the increased expression in Tetrahymena phagosomes of two genes belonging to the arginine-dependent acid tolerance pathway, we investigated the effect of $S$. Typhimurium's passage through Tetrahymena on its subsequent acid resistance while in fecal pellets. S. enterica gains resistance to acid stress at $\mathrm{pH} 3-4$ after adaptation to mild acidic conditions of $\mathrm{pH}$ 4.5-5.8 (Foster, 1995; Audia et al., 2001; Audia and Foster, 2003). The digestive process in Tetrahymena pyriformis involves a decrease in vacuolar $\mathrm{pH}$ to 5.5-6.0 after 5 min, eventually reaching 3.5-4.0 after $1 \mathrm{~h}$ (Nilsson, 1977). The viability of $S$. Typhimurium cells exposed to $\mathrm{pH} 3.4$ was enhanced in Tetrahymena fecal pellets compared with that of non-ingested cells in the same suspension. This suggested that the pathogen adapted to acidic conditions during its passage through Tetrahymena and thereby gained long-term protection from acidic stress in the external environment. The deletion of adiAY, which are part of the arginine decarboxylase system for extreme acid resistance in $S$. enterica and function under acidic $\mathrm{pH}$ in anaerobic environments (Kieboom and Abee, 2006; Alvarez-Ordonez et al., 2010), significantly decreased the viability of the pathogen in Tetrahymena. It is noteworthy that adi $Y$ is upregulated also under the acidic $\mathrm{pH}$ of macrophages (Eriksson et al., 2003). Viability of the $\triangle$ adiAY mutant in the pellets was partially restored by complementation, supporting a role for this acid stress response in the pathogen's resistance to digestion by Tetrahymena and providing further evidence for the presence of anaerobic conditions in Tetrahymena food vacuoles.

We have previously reported that ciliated protozoa isolated from bagged leafy vegetables sold at the marketplace can release viable $S$. enterica cells in pellets in vitro and that Tetrahymena has the ability to produce such pellets while grazing on $S$. enterica inoculated onto plants in the laboratory (Gourabathini et al., 2008). As the postprandial $\mathrm{pH}$ in the stomach ranges from 2.5 to 4.9 (Simonian et al., 2005), a gain in acid resistance of $S$. enterica by means of passage through Tetrahymena and egestion in its fecal pellets may enhance its survival in the human host. Hence, adaptation to low $\mathrm{pH}$ and to a range of other stresses, as shown by transcriptional profiling, may mediate the passage of this food-borne pathogen through Tetrahymena and thereby contribute to its contamination cycle.

\section{Acknowledgements}

We thank Steven Huynh for technical assistance. This work was supported by CSREES-NRI Grant 2007-03116 and by funds from USDA ARS CRIS projects 5325-42000044-00D and 5325-42000-045-00D.

\section{References}

Abu Kwaik Y, Gao LY, Stone BJ, Venkataraman C, Harb OS. (1998). Invasion of protozoa by Legionella pneumophila and its role in bacterial ecology and pathogenesis. Appl Environ Microbiol 64: 3127-3133.

Alix E, Blanc-Potard AB. (2007). MgtC: a key player in intramacrophage survival. Trends Microbiol 15: $252-256$.

Alvarez-Ordonez A, Fernandez A, Bernardo A, Lopez M. (2010). Arginine and lysine decarboxylases and the acid tolerance response of Salmonella Typhimurium. Int J Food Microbiol 136: 278-282.

Audia JP, Foster JW. (2003). Acid shock accumulation of sigma S in Salmonella enterica involves increased translation, not regulated degradation. J Mol Microbiol Biotechnol 5: 17-28.

Audia JP, Webb CC, Foster JW. (2001). Breaking through the acid barrier: an orchestrated response to proton stress by enteric bacteria. Int J Med Microbiol 291: 97-106.

Bader MW, Sanowar S, Daley ME, Schneider AR, Cho U, $\mathrm{Xu} \mathrm{W}$ et al. (2005). Recognition of antimicrobial peptides by a bacterial sensor kinase. Cell 122: $461-472$.

Balaji B, O’Connor K, Lucas JR, Anderson JM, Csonka LN. (2005). Timing of induction of osmotically controlled genes in Salmonella enterica serovar Typhimurium, determined with quantitative real-time reverse transcription-PCR. Appl Environ Microbiol 71: 8273-8283. 
Barker J, Brown MRW. (1994). Trojan horses of the microbial world: protozoa and the survival of bacterial pathogens in the environment. Microbiology 240: 1253-1259.

Bishop RE. (2005). The lipid A palmitoyltransferase PagP: molecular mechanisms and role in bacterial pathogenesis. Mol Microbiol 57: 900-912.

Brandl MT, Rosenthal BM, Haxo AF, Berk SG. (2005). Enhanced survival of Salmonella enterica in vesicles released by a soilborne Tetrahymena species. Appl Environ Microbiol 71: 1562-1569.

Brodsky IE, Ghori N, Falkow S, Monack D. (2005). Mig-14 is an inner membrane-associated protein that promotes Salmonella typhimurium resistance to CRAMP, survival within activated macrophages and persistent infection. Mol Microbiol 55: 954-972.

Cirillo JD, Falkow S, Tompkins LS. (1994). Growth of Legionella pneumophila in Acanthamoeba castellanii enhances invasion. Infect Immun 62: 3254-3261.

Cirillo JD, Falkow S, Tompkins LS, Bermudez LE. (1997). Interaction of Mycobacterium avium with environmental amoebae enhances virulence. Infect Immun 65: 3759-3767.

Cosson P, Soldati T. (2008). Eat, kill or die: when amoeba meets bacteria. Curr Opin Microbiol 11: 271-276.

Datsenko KA, Wanner BL. (2000). One-step inactivation of chromosomal genes in Escherichia coli K-12 using PCR products. Proc Natl Acad Sci USA 97: 6640-6645.

Eriksson S, Lucchini S, Thompson A, Rhen M, Hinton JC. (2003). Unravelling the biology of macrophage infection by gene expression profiling of intracellular Salmonella enterica. Mol Microbiol 47: 103-118.

Essig A, Heinemann M, Simnacher U, Marre R. (1997). Infection of Acanthamoeba castellanii by Chlamydia pneumoniae. Appl Environ Microbiol 63: 1396-1399.

Fass E, Groisman EA. (2009). Control of Salmonella pathogenicity island-2 gene expression. Curr Opin Microbiol 12: 199-204.

Faucher SP, Porwollik S, Dozois CM, McClelland M, Daigle F. (2006). Transcriptome of Salmonella enterica serovar Typhi within macrophages revealed through the selective capture of transcribed sequences. Proc Natl Acad Sci USA 103: 1906-1911.

Feng Y, Hsiao YH, Chen HL, Chu C, Tang P, Chiu CH. (2009). Apoptosis-like cell death induced by Salmonella in Acanthamoeba rhysodes. Genomics 94: 132-137.

Fok AK, Allen RD. (1975). Cytochemical localization of peroxisomes in Tetrahymena pyriformis. J Histochem Cytochem 23: 599-606.

Foster JW. (1995). Low $\mathrm{pH}$ adaptation and the acid tolerance response of Salmonella typhimurium. Crit Rev Microbiol 21: 215-237.

Gaze WH, Burroughs N, Gallagher MP, Wellington EM. (2003). Interactions between Salmonella typhimurium and Acanthamoeba polyphaga, and observation of a new mode of intracellular growth within contractile vacuoles. Microb Ecol 46: 358-369.

Gil F, Ipinza F, Fuentes J, Fumeron R, Villarreal JM, Aspee A et al. (2007). The ompW (porin) gene mediates methyl viologen (paraquat) efflux in Salmonella enterica serovar Typhimurium. Res Microbiol 158: 529-536.

Gourabathini P, Brandl MT, Redding KS, Gunderson JH, Berk SG. (2008). Interactions between food-borne pathogens and protozoa isolated from lettuce and spinach. Appl Environ Microbiol 74: 2518-2525.
Groisman EA. (2001). The pleiotropic two-component regulatory system PhoP-PhoQ. J Bacteriol 183: 1835-1842.

Gunzel D, Kucharski LM, Kehres DG, Romero MF, Maguire ME. (2006). The MgtC virulence factor of Salmonella enterica serovar Typhimurium activates $\mathrm{Na}+, \mathrm{K}+$ ATPase. J Bacteriol 188: 5586-5594.

Hahn MW, Hofle MG. (2001). Grazing of protozoa and its effect on populations of aquatic bacteria. FEMS Microbiol Ecol 35: 113-121.

Hautefort I, Thompson A, Eriksson-Ygberg S, Parker ML, Lucchini S, Danino V et al. (2008). During infection of epithelial cells Salmonella enterica serovar Typhimurium undergoes a time-dependent transcriptional adaptation that results in simultaneous expression of three type 3 secretion systems. Cell Microbiol 10: 958-984.

Hoiseth SK, Stocker BA. (1981). Aromatic-dependent Salmonella typhimurium are non-virulent and effective as live vaccines. Nature 291:238-239.

Jacobs ME, DeSouza LV, Samaranayake H, Pearlman RE, Siu KW, Klobutcher LA. (2006). The Tetrahymena thermophila phagosome proteome. Eukaryot Cell 5: 1990-2000.

Kieboom J, Abee T. (2006). Arginine-dependent acid resistance in Salmonella enterica serovar Typhimurium. J Bacteriol 188: 5650-5653.

Kovach ME, Elzer PH, Hill DS, Robertson GT, Farris MA, Roop RM et al. (1995). Four new derivatives of the broad-host-range cloning vector pBBR1MCS, carrying different antibiotic-resistance cassettes. Gene 166: 175-176.

Kyle JL, Parker CT, Goudeau D, Brandl MT. (2010). Transcriptome analysis of Escherichia coli O157:H7 exposed to lysates of lettuce leaves. Appl Environ Microbiol 76: 1375-1387.

Lock R, Öhman L, Dahlgren C. (1987). Phagocytic recognition mechanisms in human granulocytes and Acanthamoeba castellanii using type 1 fimbriated Escherichia coli as phagocytic prey. FEMS Microbiol Lett 44: 135-140.

Ly TM, Muller HE. (1990). Ingested Listeria monocytogenes survive and multiply in protozoa. J Med Microbiol 33: 51-54.

Maier RJ, Olczak A, Maier S, Soni S, Gunn J. (2004). Respiratory hydrogen use by Salmonella enterica serovar Typhimurium is essential for virulence. Infect Immun 72: 6294-6299.

Matz C, Kjelleberg S. (2005). Off the hook-how bacteria survive protozoan grazing. Trends Microbiol 13: 302-307.

Matz C, Moreno AM, Alhede M, Manefield M, Hauser AR, Givskov M et al. (2008). Pseudomonas aeruginosa uses type III secretion system to kill biofilm associated amoebae. ISME J 2: 843-852.

Miller SI, Kukral AM, Mekalanos JJ. (1989). A twocomponent regulatory system (phoP phoQ) controls Salmonella typhimurium virulence. Proc Natl Acad Sci USA 86: 5054-5058.

Molmeret M, Horn M, Wagner M, Santic M, Abu Kwaik Y. (2005). Amoebae as training grounds for intracellular bacterial pathogens. Appl Environ Microbiol 71: 20-28.

Nilsson JR. (1977). On food vacuoles in Tetrahymena pyriformis GL. J Euk Microbiol 24: 502-507.

Pace ML. (1988). Bacterial mortality and the fate of bacterial production. Hydrobiologia 159: 41-49.

Prost LR, Daley ME, Le Sage V, Bader MW, Le Moual H, Klevit RE et al. (2007). Activation of the bacterial 
sensor kinase PhoP by acidic pH. Mol Cell 26: 165-174.

Rang C, Alix E, Felix C, Heitz A, Tasse L, Blanc-Potard AB. (2007). Dual role of the MgtC virulence factor in host and non-host environments. Mol Microbiol 63: $605-622$.

Richard DJ, Sawers G, Sargent F, McWalter L, Boxer DH. (1999). Transcriptional regulation in response to oxygen and nitrate of the operons encoding the (NiFe) hydrogenases 1 and 2 of Escherichia coli. Microbiology 145(Part 10): 2903-2912.

Santiviago CA, Fuentes JA, Bueno SM, Trombert AN, Hildago AA, Socias LT et al. (2002). The Salmonella enterica sv. Typhimurium smvA, yddG and ompD (porin) genes are required for the efficient efflux of methyl viologen. Mol Microbiol 46: 687-698.

Simonian HP, Vo L, Doma S, Fisher RS, Parkman HP. (2005). Regional postprandial differences in $\mathrm{pH}$ within the stomach and gastroesophageal junction. Dig Dis Sci 50: 2276-2285.

Tezcan-Merdol D, Ljungstrom M, Winiecka-Krusnell J, Linder E, Engstrand L, Rhen M. (2004). Uptake and replication of Salmonella enterica in Acanthamoeba rhysodes. Appl Environ Microbiol 70: 3706-3714.

Vignais PM, Colbeau A. (2004). Molecular biology of microbial hydrogenases. Curr Issues Mol Biol 6: 159-188.

Yu XJ, McGourty K, Liu M, Unsworth KE, Holden DW. (2010). pH sensing by intracellular Salmonella induces effector translocation. Science 328: 1040-1043.

Zbell AL, Benoit SL, Maier RJ. (2007). Differential expression of $\mathrm{NiFe}$ uptake-type hydrogenase genes in Salmonella enterica serovar Typhimurium. Microbiology 153: 3508-3516.

Zbell AL, Maier RJ. (2009). Role of the Hya hydrogenase in recycling of anaerobically produced $\mathrm{H} 2$ in Salmonella enterica serovar Typhimurium. Appl Environ Microbiol 75: 1456-1459.

Supplementary Information accompanies the paper on The ISME Journal website (http://www.nature.com/ismej) 